\title{
Evolutionary regain of lost gene circuit function
}

\section{Journal Article}

Author(s):

Gouda, Mirna K.; Manhart, Michael (D); Balázsi, Gábor

Publication date:

2019-12-10

Permanent link:

https://doi.org/10.3929/ethz-b-000386776

\section{Rights / license:}

Creative Commons Attribution-NonCommercial-NoDerivatives 4.0 International

\section{Originally published in:}

Proceedings of the National Academy of Sciences of the United States of America 116(50), https://doi.org/10.1073/ pnas.1912257116

\section{Funding acknowledgement:}

180147 - Molecular mechanisms and evolutionary consequences of pleiotropy and single-cell variation in microbial growth (SNF) 


\title{
Evolutionary regain of lost gene circuit function
}

\author{
Mirna Kheir Gouda ${ }^{a, b}$, Michael Manhartc, and Gábor Balázsia,b,1 \\ ${ }^{a}$ The Louis and Beatrice Laufer Center for Physical and Quantitative Biology, Stony Brook University, Stony Brook, NY 11794-5252; ${ }^{b}$ Department of \\ Biomedical Engineering, Stony Brook University, Stony Brook, NY 11794-5281; and 'Institute of Integrative Biology, Eidgenössische Technische Hochschule \\ Zürich, 8092 Zürich, Switzerland
}

Edited by James J. Collins, Massachusetts Institute of Technology, Boston, MA, and approved October 30, 2019 (received for review July 17, 2019)

\begin{abstract}
Evolutionary reversibility-the ability to regain a lost function-is an important problem both in evolutionary and synthetic biology, where repairing natural or synthetic systems broken by evolutionary processes may be valuable. Here, we use a synthetic positivefeedback (PF) gene circuit integrated into haploid Saccharomyces cerevisiae cells to test if the population can restore lost PF function. In previous evolution experiments, mutations in a gene eliminated the fitness costs of PF activation. Since PF activation also provides drug resistance, exposing such compromised or broken mutants to both drug and inducer should create selection pressure to regain drug resistance and possibly PF function. Indeed, evolving $7 \mathrm{PF}$ mutant strains in the presence of drug revealed 3 adaptation scenarios through genomic, PF-external mutations that elevate PF basal expression, possibly by affecting transcription, translation, degradation, and other fundamental cellular processes. Nonfunctional mutants gained drug resistance without ever developing high expression, while quasifunctional and dysfunctional PF mutants developed high expression nongenetically, which then diminished, although more slowly for dysfunctional mutants where revertant clones arose. These results highlight how intracellular context, such as the growth rate, can affect regulatory network dynamics and evolutionary dynamics, which has important consequences for understanding the evolution of drug resistance and developing future synthetic biology applications.
\end{abstract}

evolution | gene circuit | reversal | loss of function | bistability

$\mathbf{T}$ wo ways for cells to survive stress and buy time until beneficial genetic alterations arise are through sensing and responding or through bet-hedging $(1,2)$. Gene regulatory networks evolve to provide cells with sufficient stress-protective gene expression according to these strategies. While stressprotective mutations improve the chance of survival, a tradeoff often exists between the cost and benefit of such protective mechanisms (3). For example, the expression of stress-protective genes can have a net cost in the absence of stress or even in stress if expression surpasses the levels necessary for survival (4-7). Consequently, protective but costly gene function tends to diminish or vanish from the population in the absence of stress (5, $8-10)$. How it might reappear again (evolutionary "reversal") when the stress resumes $(11,12)$ is poorly understood, specifically for gene regulatory networks that lost their costly activity. Indeed, loss-of-function mutations occur widely in laboratory evolution experiments $(5,8,13-16)$, suggesting this is a common mode of adaptation to a new environment. However, few experiments have tested how such lost functions could be restored.

Besides experimental studies of natural gene-network evolution under controlled conditions $(5,8,17-21)$, synthetic gene circuits can serve as well-characterized models of natural stressresponse modules in evolution experiments $(9,22,23)$. Wellcontrolled and tunable synthetic gene circuits that interact minimally with the host genome (24-27) can aid the interpretation of experimental outcomes. Considering future applications of synthetic biology $(28,29)$, it is important to explore potential evolutionary strategies that can restore synthetic gene circuit function if it happens to break over time $(9,30,31)$, without circuit reintroduction or repair by rational means or mutagenesis (32). Directed evolution studies have improved enzymes or metabolic pathways $(33,34)$, but only through mutagenesis in single proteins, leaving it unclear whether noncoding or genomic mutations could improve or restore gene-network performance. Adaptation under function-restoring selection pressure allowing host genome changes could reveal essential parameters and new methods to design and improve engineered cell fitness and robustness in various growth conditions.

To understand how a network that lost its costly activity in the absence of stress adapts and possibly regains function in the presence of stress, here we used previously evolved, broken versions of a synthetic "positive feedback" (PF) gene circuit originally integrated into the haploid Saccharomyces cerevisiae YPH500 genome (6). Many different reverse tetracycline Transcriptional Activator (rtTA) mutants arose in previous evolution experiments (9), apparently eliminating costly rtTA function in the absence of antibiotic stress. We evolved 7 such broken PF mutants in both inducer and antibiotic, where regaining rtTA function should be beneficial. By examining the phenotypic and genetic changes through fluorescence and fitness measurements, as well as sequencing, we observed 3 different classes of evolutionary dynamics, depending on whether ancestral mutants were quasifunctional, dysfunctional, or nonfunctional. In quasifunctional mutants, slow growth from drug exposure initially enriched the high-expressor subpopulation, but then new drug-resistance mutations slightly elevated basal expression, eliminating the benefit of high expression and diminishing the high-expressor fraction through a growth-dependent shift in dynamics. Nonfunctional mutants acquired new drug-resistance

\section{Significance}

Natural or synthetic genetic modules can lose their function over long-term evolution if the function is costly. How populations can evolve to restore such broken function is poorly understood. To test the reversibility of evolutionary breakdown, we use yeast cell populations with a chromosomally integrated synthetic gene circuit. In previous evolution experiments the gene circuit lost its costly function through various mutations. By exposing such mutant populations to conditions where regaining gene circuit function would be beneficial, we find adaptation scenarios with or without repairing lost gene circuit function. These results are important for drug resistance or future synthetic biology applications where evolutionary loss and regain of function play a significant role.

Author contributions: M.K.G. and G.B. designed research; M.K.G. and G.B. performed research; M.K.G. contributed new reagents/analytic tools; M.K.G., M.M., and G.B. analyzed data; and M.K.G. and G.B. wrote the paper.

The authors declare no competing interest.

This article is a PNAS Direct Submission.

This open access article is distributed under Creative Commons Attribution-NonCommercialNoDerivatives License 4.0 (CC BY-NC-ND)

Data deposition: Data and Matlab code associated with the figures can be found at https://drive.google.com/drive/folders/13jrR8XHP4p96-bcNGTlxRpFzjTfRvPkh and https:// drive.google.com/drive/folders/1sZxfXY-AzigYSCDhX_SW0bSNqfgo72lh.

${ }^{1}$ To whom correspondence may be addressed. Email: gabor.balazsi@stonybrook.edu.

This article contains supporting information online at https://www.pnas.org/lookup/suppl/ doi:10.1073/pnas.1912257116/-/DCSupplemental.

First published November 21, 2019. 
mutations that slightly elevated basal expression and never developed high expression. Finally, the dysfunctional mutant populations evolved similarly to quasifunctional mutants, but more slowly and gave rise to clones with repaired network function. Overall, we found numerous extracircuit mutations, but no novel coding-sequence mutations inside the gene circuit directly related to these expression changes. Detected genomic mutations possibly affect important cellular processes, such as transcription, translation, macromolecule degradation, and others. Our findings provide insights into the evolutionary reactivation of broken network modules, depending on their dynamics as well as the costs and benefits after the stress recurs.

\section{Results}

Hyperinduction and Slow Growth Reveal 3 Classes of rtTA Mutations. The original PF gene circuit (6) consists of a doxycycline (Dox)inducible rtTA transcriptional activator that identically upregulates both its own expression and the expression of the Zeocin resistance gene, zeoR, fused to yEGFP (yEGFP::zeoR), by binding to 2 tet $O 2$ operator sites upstream of the $r t T A$ and the $y E G F P:: z e o R$ coding regions (Fig. $1 A$ ). The yEGFP::ZeoR bifunctional fusion protein (35) precisely reports ZeoR levels and protects cells by directly binding to Zeocin (36) to prevent its intercalation into DNA, which causes DNA breaks and thus cell cycle arrest or death (37). Previously, we evolved yeast populations carrying the PF circuit (9) in $2 \mu \mathrm{g} / \mathrm{mL}$ of Dox inducer without Zeocin antibiotic, a condition denoted "D2Z0," where the first number represents Dox concentration in micrograms per milliliter, while the second number represents Zeocin concentration in milligrams per milliliter. Since the activation of the PF stress-response module is costly in the absence of stress, the rtTA protein apparently lost its function during these evolution experiments through different coding-sequence mutations (9). To test whether evolutionary repair of lost rtTA function was possible, we further evolved 7 of these loss-of-function rtTA mutants from the previous experiment (Fig. $1 B$ ) in the condition D2Z2 $(2 \mu \mathrm{g} / \mathrm{mL}$ Dox and $2 \mathrm{mg} / \mathrm{mL}$ Zeocin $)$, which should activate the original PF gene circuit or any reverting mutants, ensuring their survival in stress. We will refer to the 7 ancestral mutations by their genotype: "Missense 1, 2, 3, and 4" correspond to $\mathrm{rtTA}_{+189 \mathrm{C} \rightarrow \mathrm{G}}, \mathrm{rtTA}_{+562 \mathrm{~T} \rightarrow \mathrm{C}}, \mathrm{rtTA}_{+275 \mathrm{G} \rightarrow \mathrm{A}}$, and $\mathrm{rtTA}_{+13 \mathrm{G} \rightarrow \mathrm{T}}$, respectively; "Nonsense" corresponds to $\mathrm{rtTA}_{+442 \mathrm{G} \rightarrow \mathrm{T}}$; "Duplication" corresponds to rtTA $+95,30 \mathrm{bp}$; and "Deletion" corresponds to $\mathrm{rtTA}_{+651,78 \mathrm{bp} \Delta}$ (SI Appendix, Table S1).

To understand the dynamics of the original PF gene circuit (Fig. $1 A$ ) and its 7 rtTA mutants (Fig. $1 B$ ), we studied qualitatively a simple mathematical model of this genetic autoregulatory module (SI Appendix, Mathematical Modeling). In ratebalance plots from these models (Fig. $1 C$ ), intersections between rtTA synthesis (Fig. $1 C$, blue sigmoid) and loss (Fig. $1 C$, red elbow) rate curves correspond to stable (Fig. $1 C$, full circles) and unstable (Fig. $1 C$, open circles) $r t T A$ gene expression states that impose corresponding cellular yEGFP::zeoR protein levels. For example, the original PF gene circuit (6) is monostable below a threshold
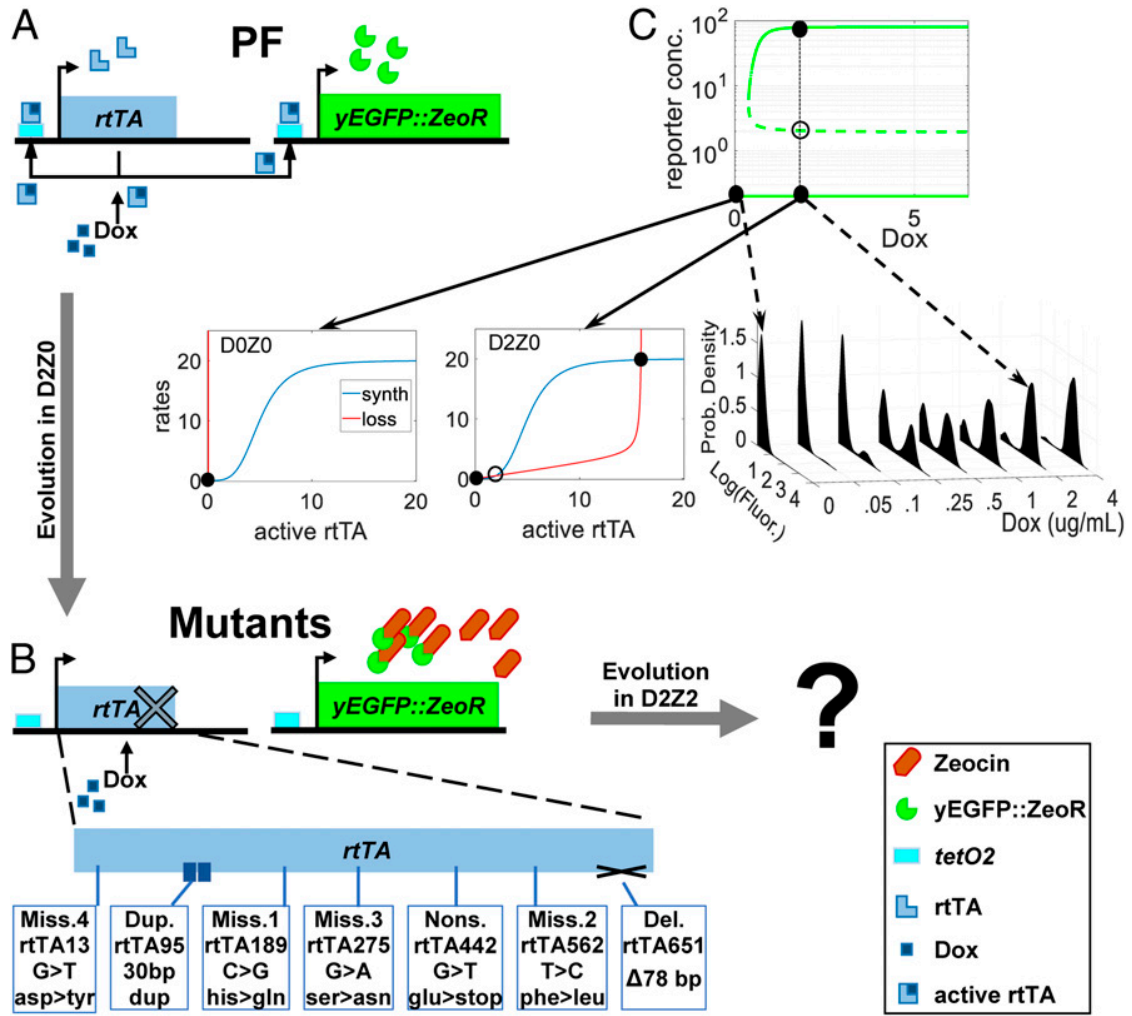

Fig. 1. The PF gene circuit lost bistability and costly rtTA function by multiple mutations. $(A)$ In the original PF gene circuit, the inducer Dox binds and activates the rtTA protein, which identically activates its own rtTA gene and yEGFP::zeoR. Since rtTA activity is costly, loss of rtTA function is evolutionarily beneficial in D2ZO. (B) We selected 7 mutants that arose in D2Z0, each improving fitness by PF breakdown. Now we evolve each mutant in D2Z2 where regaining rtTA function would be beneficial. DxZy denotes the concentrations of the inducer Dox and the antibiotic Zeocin in micrograms per milliliter and milligrams per milliliter, respectively. $(C)$ The original PF gene circuit undergoes a saddle-node bifurcation, changing from monostable to bistable dynamics when Dox exceeds a threshold. The top graph shows yEGFP::zeoR levels versus Dox from a simple mathematical model (SI Appendix, Mathematical Modeling). The blue and red curves on the bottom graphs represent inactive rtTA synthesis and loss rates; while filled and open circles denote stable and unstable steady states, respectively. The active rtTA levels corresponding to the circles impose the yEGFP::zeoR levels on the top graph and on the right, where experimental yEGFP::zeoR expression histograms versus Dox demonstrate the bifurcation. 
concentration of $\sim 0.05 \mu \mathrm{g} / \mathrm{mL}$ Dox, with a single intersection corresponding to a single low rtTA expression state imposing low yEGFP::zeoR expression. As Dox concentrations increase, the elbow-shaped curve of Dox-free, inactive rtTA loss (from combined dilution and degradation) moves rightward, approaching the sigmoidal rtTA synthesis curve until they meet and intersect 3 times. Thus, for Dox concentrations above the threshold, a second stable high-expression state emerges, indicating a transition from monostable to bistable dynamics through a saddle-node bifurcation (Fig. $1 C$ ). Losing the high geneexpression peak during long-term evolution in D2Z0 (9) could indicate either that a mutant gene circuit became completely noninducible, or that the sigmoidal rtTA synthesis rate curve shifted somehow, elevating the bistability threshold beyond D2Z0. Still, the elbow-shaped loss curve slides rightward as Dox increases further, so it could still reach and intersect a rightshifted sigmoid curve 3 times, causing a high-expression peak to still emerge at sufficiently high Dox concentrations. To examine the possibility of such weaker but still present rtTA function, we looked for high expressors in flow cytometry histograms of each mutant in D2Z0. Indeed, upon closer examination, we noticed $\sim 1 \%$ high-expressing cells in Missense 1 and 2 populations, but not any other mutants (SI Appendix, Figs. S3-S8). Therefore, we deem the Missense 1 and 2 mutant gene circuits to be quasifunctional rather than completely nonfunctional ( $S I$ Appendix, Table S1).

To fully test which of the 7 loss-of-function PF mutants are still quasifunctional, we next hyperinduced them with excess Dox. We expected that hyperinduction would shift the elbow-shaped curve of rtTA loss farther rightward and move quasifunctional rtTA mutants into their bistable regime (Fig. $1 C$ and SI Appendix, Mathematical Modeling), thus generating high-expressor subpopulations, while nonfunctional mutants would remain unimodal. To this end we grew clonal populations carrying each rtTA mutation in D6Z0 (Fig. $2 A$ and $B$ ), a 3-fold higher $(6 \mu \mathrm{g} / \mathrm{mL})$
Dox concentration than D2Z0 where they previously arose. These hyperinducing conditions confirmed that Missense 1 and 2 were indeed quasifunctional, while the remaining 5 mutantsMissense 3 and 4, Nonsense, Duplication, and Deletion-still appeared uninducible and thus nonfunctional (SI Appendix, Table S1). Excess Dox did not alter the growth rate of any clones, indicating that hyperinduction is not toxic for any mutant. Thus, any further mutations abolishing leftover rtTA function in Missense 1 and 2 would not be beneficial. The results did not change upon hyperinducing the mutants in $8 \mu \mathrm{g} / \mathrm{mL}$ Dox (SI Appendix, Fig. S1 $A$ ). Hysteresis experiments also confirmed the upshift of bistability range for Missense 1 and 2 compared to PF (SI Appendix, Fig. S2).

Mathematical models suggested another mechanism besides hyperinduction that can cause stable high expression. Slow growth reduces dilution of cell contents and thus tilts downward the rtTA loss curve, besides shifting it rightward. This moves the quasifunctional PF mutants into the bistable regime (SI Appendix, Mathematical Modeling), similar to growth-mediated bistability observed in other systems $(38,39)$. To experimentally test this, we grew PF mutants as well as standard PF cells in D2Z0 with $7.5 \%$ ethanol that slowed the growth rate to a value similar to that in D2Z2 (SI Appendix, Figs. S3-S8). Interestingly, ethanol strongly enriched the high-expressor fraction of ancestral PF cells, from 71 to $91 \%$ in D2Z0. As expected, slow growth due to ethanol also enriched the high-expressor fractions of Missense 1 (more than 2-fold) and Missense 2 (about 9-fold). Most surprisingly, ethanol caused a few high-expressor cells to appear even in Missense 3, which failed to respond to hyperinduction in D6Z0 or D8Z0. Therefore, we classify Missense 3 as a dysfunctional mutant (SI Appendix, Table S1), unlike the nonfunctional mutants Nonsense and Deletion, which do not become bistable even in slow growth. We obtained comparable results using Cisplatin, which interferes with DNA (40) like Zeocin (37). In contrast, G418 did not have this effect,

\section{A Quasi-functional}
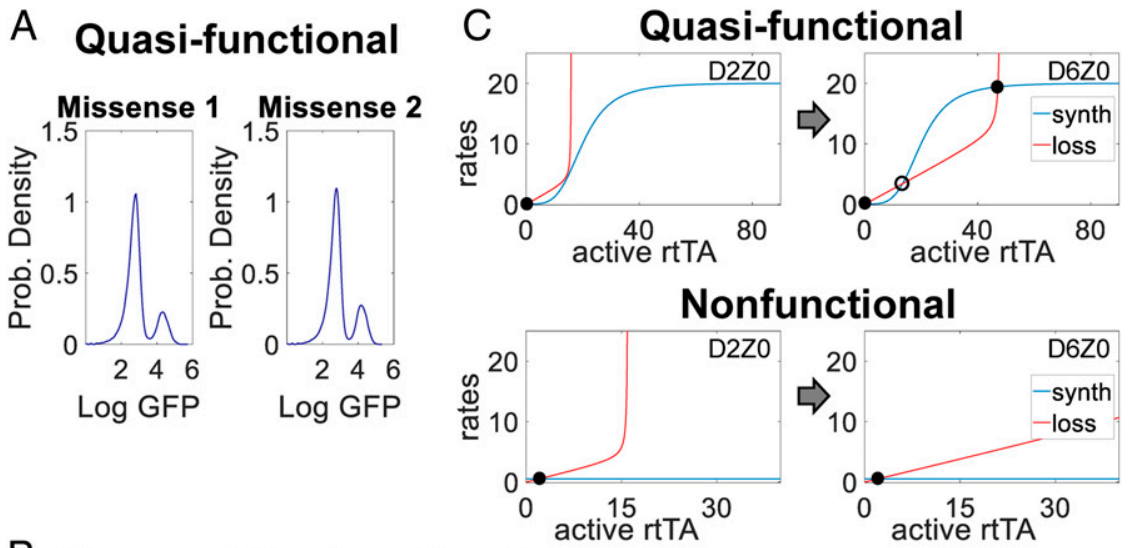

$B$ Dys-and Nonfunctional
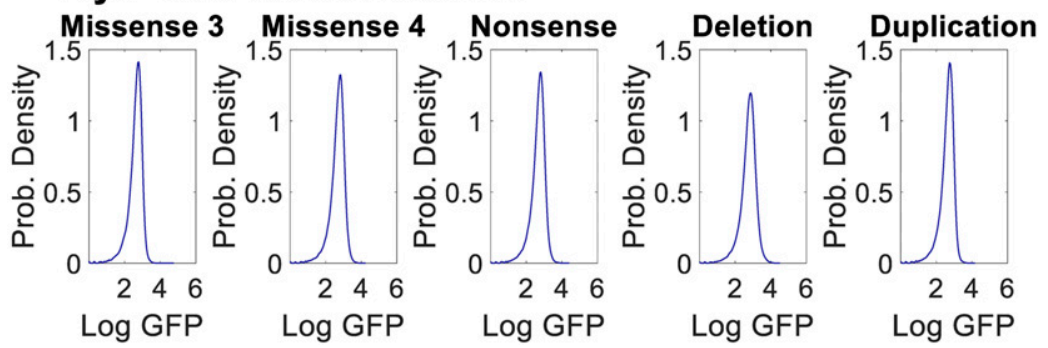

Fig. 2. Quasifunctional rtTA mutants revealed by hyperinduction. ( $A$ and $B$ ) Gene-expression histograms of quasifunctional, dysfunctional, and nonfunctional PF mutants hyperinduced in D6Z0 (24 h). A high-expression peak in this condition indicates bistabiltiy. (C) Plots illustrating the dynamical effect of hyperinduction on quasifunctional and nonfunctional PF mutants. Filled and open circles denote stable and unstable steady states, respectively. 
presumably because protein synthesis inhibition (41) abolishes gene circuit function (SI Appendix, Figs. S3-S8). Overall, these data and the model indicate that slow growth due to stressors can cause shifts in dynamics that generate high expressors in Missense 3 and can enrich the already present high expressor subpopulations of Missense 1 and 2.

Evolution Does Not Revert Quasifunctional Mutants. Next, we set out to test if evolution could revert the 2 quasifunctional mutants (Missense 1 and 2) to regain stronger rtTA function. Thus, we evolved 3 replicates each of Missense 1 and 2 in D2Z2 medium, where regaining high rtTA activity would beneficially shift cells into the drug-resistant state of high yEGFP::ZeoR expression. For control purposes, we also propagated the same initial populations in D2Z0.

We observed qualitatively similar evolutionary dynamics for both Missense 1 and 2 (Fig. 3), which started with a minimal $(0.47 \%$ and $0.63 \%)$ subpopulation of high-expressor cells immediately upon transfer from D2Z0 into D2Z2. Early on, population fitness levels dropped significantly in all D2Z2 cultures compared to the control D2Z0 cultures (Fig. $3 C$ and $D$ ). Soon afterward, drug exposure generated a substantial high-expressing subpopulation for a few days (Fig. $3 A$ and $B$ ). Growth rates started recovering in $\sim 4 \mathrm{~d}$, as the high-expressor peak increased (Fig. $3 C$ and $D$ ). From day 1 onward, we also observed a slight upward fluorescence shift for low expressors, corresponding to elevated basal yEGFP::ZeoR levels compared to D2Z0 controls (Fig. $3 A$ and $B$ ). This slight upward expression shift is clearly distinguishable from "induced" high expression and resembles phenotypes arisen previously during evolution in Zeocin drug (D0Z2) (9), where various extracircuit mutations, often coupled with intracircuit synonymous and promoter mutations, elevated the low, basal yEGFP::ZeoR expression. Concurrently, the high- expressor population fraction reached a maximum around day 4 and then returned to the same low level as in D2Z0 within $\sim 8 \mathrm{~d}$ (Fig. $3 A$ and $B$ ).

Considering that high expressors were rare in D2Z0, while in D2Z2 their fraction initially increased and then later dropped back to the D2Z0 level, at least 2 hypotheses are possible. First, compensatory mutations in rtTA or elsewhere could improve rtTA activator function, thereby increasing the high-expressor fraction, and then later subsequent mutations could revert this effect. To identify such compensatory mutations, we performed whole-genome sequencing (WGS) on 1 replicate population each for both mutants on days 1,3 , and 14. The original rtTA mutations already present at the beginning of the experiment were identified at $100 \%$ frequency at all time points for both Missense 1 and 2. However, we identified no other intracircuit mutations. Since certain variant types (e.g., deletions and duplications) in the PF gene circuit are difficult to detect by WGS (24), we also performed Sanger sequencing of the PF region in 10 individual clones from each mutant at day 14 (SI Appendix, Table S2). All Missense 1 clones carried a deletion of the first tetO2 operator upstream of the $y E G F P:: z e o R$ coding region, whereas the other tetO2 site stayed intact. All Missense 2 clones carried only their original gene circuit mutation without any tetO2 or other circuit modifications, despite their identical phenotypes to Missense 1 . Therefore, there is no phenotypic signature directly and specifically attributable to the tetO2 operator site deletion (SI Appendix, Table S3). Overall, the only de novo intracircuit mutation we found did not explain the observed gene-expression changes.

High expression requires rtTA function. How then could a substantial high-expressor peak emerge and later diminish without any changes in the rtTA coding sequence or its promoter? Modeling and experiments suggested a second hypothesis: By
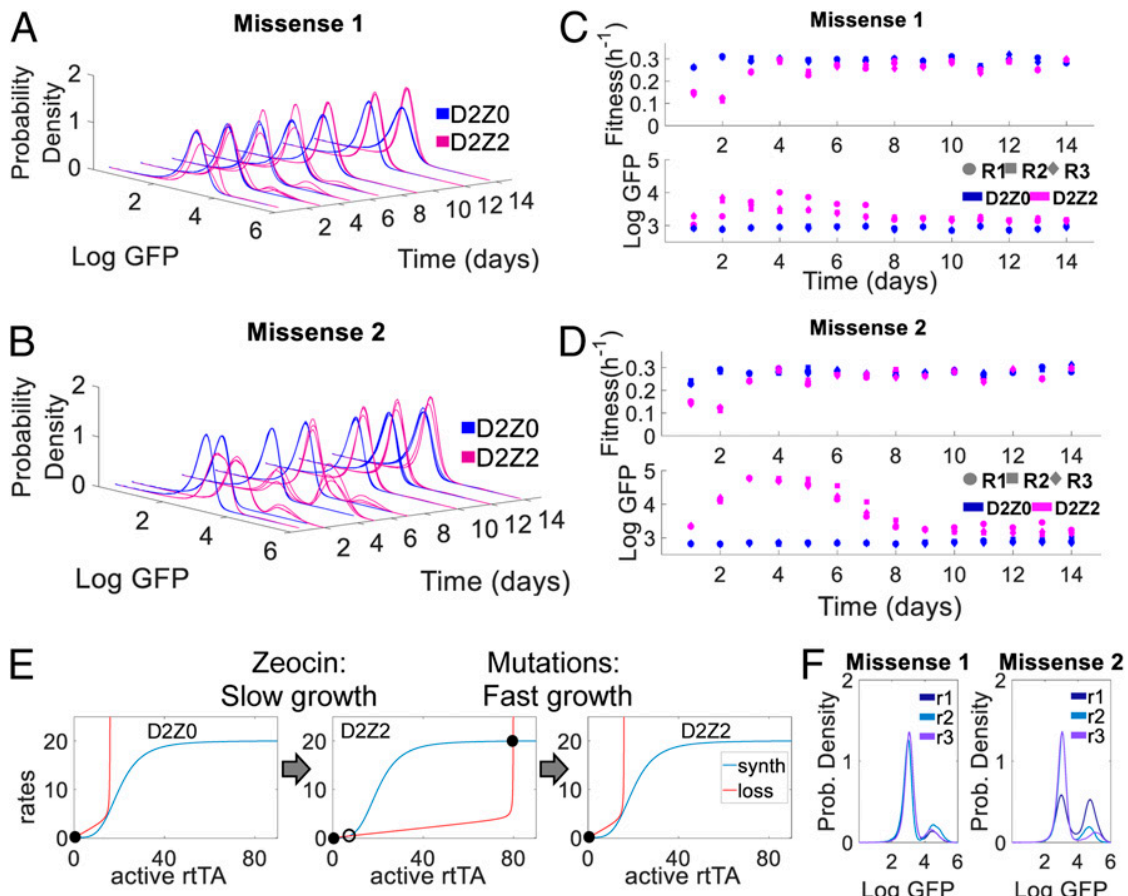

Fig. 3. Evolutionary dynamics of quasifunctional mutants. ( $A$ and $B$ ) Histogram of $y E G F P:: z e o R$ expression for Missense 1 and 2 replicates in D2Z0 (blue, control) and D2Z2 (magenta) over the course of $14 \mathrm{~d}$. ( $C$ and $D$ ) Population fitness of Missense 1 and 2 replicates in D2Z0 (blue) and D2Z2 (magenta) over the course of $14 \mathrm{~d}$. $(E)$ Changes of PF dynamics due to slower growth and selection in Zeocin, and then, reversion to normal growth in D2Z2 after the emergence of drug-resistance mutations. Filled and open circles denote stable and unstable steady states, respectively. $(F)$ Gene-expression histograms of evolved Missense 1 and 2 population replicates hyperinduced in D6Z0 at the end of experimental evolution. A high-expression peak in this condition indicates the presence of quasifunctional mutants in the population. 
decelerating growth and reducing dilution, Zeocin tilts downward and shifts rightward the red elbow curve of rtTA loss (Fig. 3E). This causes a shift in dynamics (SI Appendix, Mathematical Modeling) that enriches the rare high-expressing drug-resistant cells that preexist in D2Z0 (SI Appendix, Figs. S3 and S4), which are then further enriched by phenotypic selection. At the same time, the low-expressing subpopulation will remain drug-sensitive, enabling any mutants that elevate basal expression to grow faster and spread in the population. Once such fast-growing mutants arise, their faster growth/dilution rate tilts upward and shifts leftward the red elbow curve (Fig. $3 E$ ), causing a return of dynamics toward monostability (SI Appendix, Mathematical Modeling). Concurrently, the benefits of high-expression also vanish, causing the high-expressor fraction to diminish back to the D2Z0 levels. Importantly, these events can occur without any mutations in the PF gene circuit, as long as extracircuit mutations can raise basal yEGFP::ZeoR expression to promote fast growth in Zeocin.

To identify such extracircuit mechanisms of $y E G F P:: z e o R$ dependent Zeocin resistance, we looked for genomic, extracircuit mutations in the WGS data (Materials and Methods). In the Missense 1 population at day 14 we found moderate-frequency ( $\sim 15 \%)$, nonsynonymous mutations in 2 genes affecting protein stability and transcription, $P A H 1$ and SET3, including a 1-bp frameshift deletion in the latter. Additional mutations in other genes and intergenic regions exist at lower frequencies (SI Appendix Table S3 and Dataset S1). In contrast to Missense 1, evolved Missense 2 populations carried multiple extracircuit mutations exceeding $90 \%$ in frequency at day 14 (SI Appendix, Table S3 and Dataset S2). Mutations in the genes $P H B 2, M D M 32$, and $C O X 1$ suggest alteration in mitochondrial metabolism and function. We also observed a synonymous mutation in the FG-nucleoporin NUP159 gene, which is involved in posttranscriptional regulation (42).

To test if elevated $y E G F P:: z e o R$ gene expression mediates drug resistance without rtTA activity, we compared the growth of Missense 1- and 2-evolved populations and clonal isolates to their unevolved Missense 1 and 2 ancestors in Zeocin-only media (D0Z2). Evolved Missense 1 and 2 populations and clonal isolates grew significantly faster than the ancestral PF or unevolved Missense 1 and 2 cells in D0Z2 (SI Appendix, Figs. S12 and S13), indicating that the evolved Missense 1 and 2 populations were Zeocin-resistant independently of rtTA.

If the $r t T A$ coding sequence and PF gene circuit dynamics did not change during evolution, hyperinduction should affect Missense 1 and 2 similarly to their ancestors. To test this, we hyperinduced each final evolved Missense 1 and 2 replicate population in D6Z0. Indeed, the evolved Missense 1 and 2 populations developed high expression in D6Z0 (Fig. $3 F$ ) and in D8Z0 (SI Appendix, Fig. S1B) as their ancestors did. Similarly, hyperinduction did not affect the growth rates of the evolved populations. Overall, the Missense 1 and 2 evolution experiments indicate that slow growth and phenotypic selection initially enrich the high-expressor fraction nongenetically, but then extracircuit mutations accelerate growth by elevating basal stress-protective yEGFP::ZeoR expression, which shifts the dynamics to diminish the high-expressor fractions back to levels equivalent to $\mathrm{D} 2 \mathrm{Z} 0$.

Most Nonfunctional Mutants Never Regain rtTA Function. As the lack of high expression in D6Z0 indicates, the 4 other initial PF mutants (Missense 4, Nonsense, Duplication, and Deletion) had mutations that disrupted rtTA protein function such that it became completely uninducible, regardless of inducer amount (Fig. $2 B$ and SI Appendix, Fig. S1A) or growth rate (SI Appendix, Figs. $\mathrm{S} 6$ and S7). To test if evolution could restore rtTA function in any of these nonfunctional mutants, we also evolved 3 replicates of each in D2Z2 where regaining rtTA activity would generate a beneficial high $y E G F P:: Z e o R$ expression peak.

Early in the evolution experiment (Fig. $4 B$ and SI Appendix, Fig. S9 $A-F)$, the growth rate in $\mathrm{D} 2 \mathrm{Z} 2$ of each nonfunctional mutant population dropped significantly below the controls evolving in D2Z0. The growth rates of the Missense 4, Nonsense, and Duplication populations then recovered over $\sim 5 \mathrm{~d}$, while the growth rates of the Deletion populations recovered within $3 \mathrm{~d}$, approaching that of D2Z0 control cultures. Unlike quasifunctional mutants, nonfunctional mutants never gave rise to a highexpressor subpopulation (Fig. $4 A$ and SI Appendix, Fig. S9 $A-C$ ). The $y E G F P:: z e o R$ expression distributions remained unimodal but shifted slightly upwards compared to the basal (D2Z0 control) expression in all experimental cultures. Consequently, these evolving cell populations relied again on $y E G F P:: z e o R$ expression to gain drug resistance and increase their fitness, without repairing rtTA function. Indeed, Sanger sequencing of 10 isolated clones from each mutant revealed no additional mutations inside the gene circuit, except for the deletion of the first tetO2 site upstream of $y E G F P: \because z e o R$ in 0 of 10 Missense 4 clones, 1 of 10 Nonsense clones, 8 of 10 Deletion clones, and 2 of 10 Duplication clones (SI Appendix, Table S4). All mutants retained the original rtTA mutation and no other rtTA mutations were detected. As with Missense 1 and 2, there was no phenotype associated specifically with the tetO2 deletion (SI Appendix, Table S4).

To identify possible extracircuit mutations causing the slight basal expression increase, we analyzed the WGS data. We found mutations in or near genes controlling mRNA and protein levels through degradation or synthesis, such as the poly-A tail shortening CCR4, the TFIID subunit TAF2, the ribosomal subunit $R P L 41 A$, and others (Datasets S4-S7).

To examine rtTA nonfunctionality at the end of the evolution experiment, we hyperinduced final evolved populations in D6Z0. As expected, the yEGFP::ZeoR expression of nonfunctional mutants remained unimodal and low in D6Z0 and in D8Z0 (Fig. $4 C$ and SI Appendix, Figs. S1B and S9G), indicating that rtTA and the gene circuit in these mutants remained nonfunctional at the end of evolution in D2Z2, as anticipated.

As we did for Missense 1 and 2, we also examined rtTAindependent Zeocin resistance of nonfunctional mutants by growing evolved populations and isolated clones in D0Z2 media
A

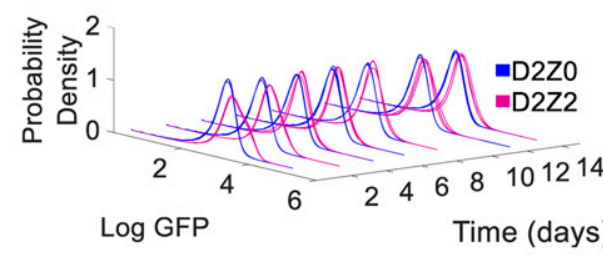

$\mathrm{B}$

$$
\text { ב्ञ }
$$
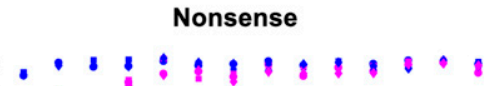

1.
ع 0

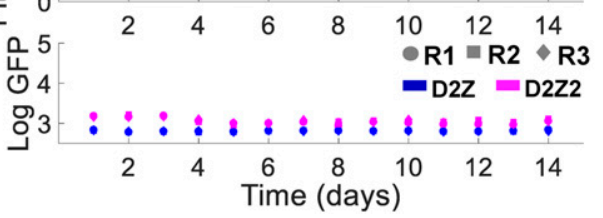

Fig. 4. Example of evolutionary dynamics of nonfunctional mutants that never regain rtTA function. (A) Histograms of $y E G F P:: z e o R$ expression of 3 replicates of Nonsense mutant in D2Z0 (blue, control) and D2Z2 (magenta) over the course of $14 \mathrm{~d}$. (B) Fitness and mean $y E G F P:: z e o R$ expression plots for Nonsense mutant computed for each day of the experiment. $(C)$ Geneexpression histograms of evolved population replicates hyperinduced in D6Z0 at the end of experimental evolution. 
for $24 \mathrm{~h}$. The evolved Missense 4, Nonsense, and Duplication populations and isolated clones grew significantly faster than their unevolved ancestors (SI Appendix, Figs. S12 and S13). Interestingly, the unevolved Deletion mutant seemed to have some preexisting yEGFP::ZeoR-dependent resistance to Zeocin (SI Appendix, Figs. S12 and S13). This corroborates the faster Deletion recovery and slightly higher yEGFP::ZeoR basal expression compared to all other nonfunctional mutants upon Zeocin exposure, already at day 1 (SI Appendix, Fig. S10), and reinforces general reliance on slightly elevated yEGFP::ZeoR expression for drug resistance.

Dysfunctional Mutants Can Regain PF Gene Circuit Function. Surprisingly, during evolution in D2Z2, all 3 replicates of the dysfunctional rtTA mutant (Missense 3) temporarily gave rise to a significant high-expressor subpopulation that persisted for over a week for each replicate. Ultimately, toward the end of the evolution experiment, these high-expressor subpopulations diminished and the fluorescence shift of the low peak indicated a slight increase in basal yEGFP::zeoR expression similar to all other genotypes. The fitness trends of each Missense 3 replicate resembled those of the other mutants: The initially low population fitness levels recovered to normal (control) levels within $\sim 4 \mathrm{~d}$ (Fig. $5 A$ and $B$ ).

Once again, despite the marked appearance and subsequent gradual disappearance of the high-expressing peak in $\mathrm{D} 2 \mathrm{Z} 2$, we found no additional rtTA coding sequence mutations in Missense 3 populations. Sanger sequencing from day 14 detected only 1 intracircuit mutation in some clones: The deletion of 1 tetO2 site upstream from yEGFP::zeoR, while the other tetO2 sites remained intact. Interestingly, all individual Missense 3 clones that acquired a tetO2 deletion had unimodal gene expression, whereas the clones that lacked this mutation were bistable in D2Z2 at the end (SI Appendix, Table S5).

Mathematical modeling (SI Appendix, Mathematical Modeling) suggested that gene circuit dysfunction in Missense 3 is due to a drop in rtTA activator capacity (Fig. 5C), rather than reduced inducer sensitivity as for Missense 1 and 2 . This means that the rtTA synthesis rate curve collapses downward (Fig. 5C) instead of shifting rightward (Fig. $2 C$ ) in such dysfunctional mutants.
Importantly, this collapse implies that the elbow-shaped rtTA loss curve will always be above the collapsed sigmoid during normal growth, so the curves will miss intersecting each other again regardless of the hyperinducing Dox level. Yet, a bistable region can still be reached if slow growth/dilution tilts downward and shifts rightward the rtTA loss curve, causing high-expressors to emerge, despite Missense 3 being completely unresponsive to hyperinduction. Indeed, adding ethanol or Cisplatin but not G418 to $\mathrm{D} 2 \mathrm{Z} 0$ indicated that slow growth enables some high expression in Missense 3 (SI Appendix, Fig. S5). Zeocin in D2Z2 enriches this high-expressor fraction further by selection. Mutations elevating the entire sigmoidal synthesis curve could then accelerate growth, thus reestablishing and maintaining bistability (Fig. 5C), while also stabilizing the high-expressor fraction (SI Appendix, Mathematial Modeling). On the other hand, not all mutations elevate expression sufficiently to maintain bistability as growth accelerates, causing the high-expressor fraction to diminish in the evolving population, which can be a mixture of such mutation types.

To identify these mutations predicted by the model, we examined WGS data for 2 replicate populations of Missense 3. Relevant high-frequency mutations potentially related to transcriptional regulation in replicate 1 (r1) included 1-bp frameshift deletions in SIF2 and SSN3, as well as a nonsynonymous mutation in SSN2. Missense 3 replicate 3 (r3), on the other hand, carried multiple genomic mutations that spread in the entire population by day 14 of the experimental evolution, including missense mutations in mediator complex components $S R B 8$ and $M E D 6$, both of which are involved in RNA polymerase IIdependent transcriptional regulation (43-45) and could elevate both basal and maximal rtTA levels (the entire rtTA synthesis curve) as the model suggested. In addition, a chaperonin subunit $C C T 7$ mutation suggests effects on general protein folding (46), and mutations in the SAP185 and EXG1 coding regions suggest altered cell cycle regulation $(47,48)$ (SI Appendix, Table S5 and Dataset S3).

We also hyperinduced the final evolved replicate populations from Missense 3 in D6Z0 to examine PF function at the end of evolution. Surprisingly, 2 evolved Missense 3 replicates ( 1 and $\mathrm{r} 3$ ) had bimodal distributions, indicating that gene circuits in some clones became quasifunctional or fully functional.
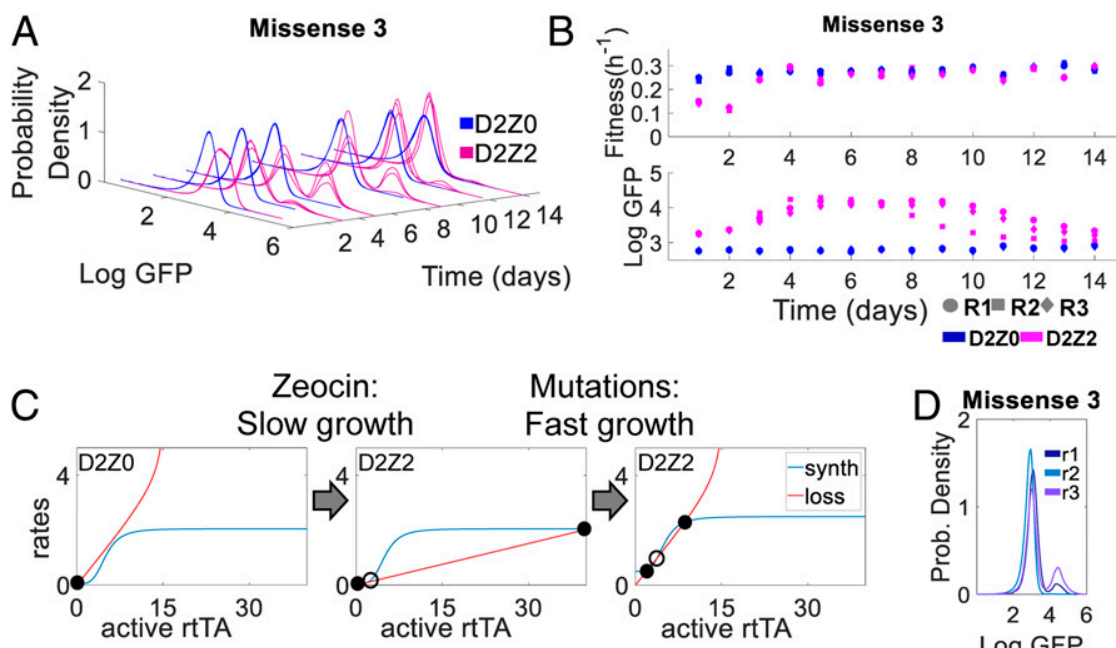

Zeocin: growth

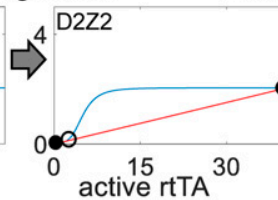

Mutations:

Fast growth

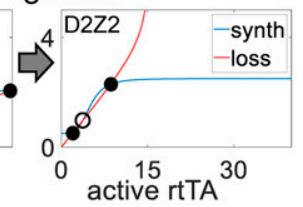

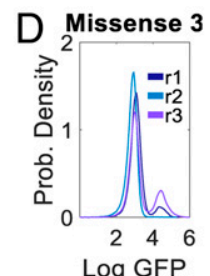

Fig. 5. Evolutionary dynamics of the dysfunctional mutant Missense 3. $(A)$ Histogram of $y E G F P:: z e o R$ expression of Missense 3 replicates in D2Z0 (blue, control) and D2Z2 (magenta) over the course of $14 \mathrm{~d}$. (B) Population fitness mean yEGFP::zeoR of Missense 3 cells in D2Z0 (blue) and D2Z2 (magenta) over the course of $14 \mathrm{~d}$. (C) Evolutionary changes in gene circuit dynamics involve slow growth-induced bistability due to Zeocin, followed by extracircuit mutations that can shift the entire rtTA synthesis curve upward, reestablishing bistability. Filled and open circles denote stable and unstable steady states, respectively. (D) Gene-expression histograms of evolved population replicates hyperinduced in D6Z0 at the end of experimental evolution. A high-expression peak in this condition indicates the presence of quasi- or fully functional revertant mutants in the population. 
Missense $\mathrm{r} 2$ had a unimodal distribution in D6Z0, indicating that it remained insensitive to hyperinduction (Fig. $5 D$ ). We observed the same in D8Z0 (SI Appendix, Fig. S1B).

Finally, as for other mutants, we assessed rtTA-dependence of Zeocin resistance in evolved Missense 3 populations and clonal isolates by culturing them in D0Z2. OD600 measurements of original and evolved populations (SI Appendix, Figs. S12 and S13) indicated that evolved Missense 3 replicate populations grew significantly faster than their unevolved ancestor. Interestingly, isolated bimodal Missense 3 clones with mutationfree gene circuits grew slower in D0Z2 than unimodal clones with a tetO2 site deletion upstream from $y E G F P:: z e o R$. This further supports that the tetO2 site deletion contributes to $r T A$ independent, but $y E G F P:: z e o R$-dependent drug resistance, whereas bimodal Missense 3 clones rely on regained rtTA activity for resistance.

Sorting Dysfunctional Mutants Yields Clones with Regained rtTA Function. So far, a likely common explanation for the observed evolutionary dynamics is the appearance and spread of extracircuit mutations that accelerate growth of the drug-sensitive, lowexpressor subpopulation by elevating yEGFP::zeoR basal expression, thereby returning the dynamics toward the unimodal low-expression regime. If this is the case, then separating the high- and low expressors around day 4 should result in different evolutionary dynamics. Since drug selects against ancestral lowexpressor cells, the increased selection pressure should cause drug-resistance mutations to spread quickly among low-sorted cells, preventing them from generating a substantial highexpressing peak. On the other hand, high-sorted cells would be insensitive to Zeocin and mutations could spread among them only after they generate a low-expressing peak by phenotypic switching. Therefore, high-sorted cells should take longer to become unimodal than low-sorted cells. Also, as discussed above, some quasi- or fully functional PF revertant clones may exist. If any revertant strains arose, we may be able to isolate them among the high-sorted cells because high expression is the hallmark of PF functionality.

To test these hypotheses, we separated the low-expressor and high-expressor subpopulations by fluorescence-activated cell sorting (FACS) at the end of day 4 of the original evolution experiment and cultured these high- and low-sorted subpopulations separately in the D2Z2 condition for $12 \mathrm{~d}$ (Fig. 6). As predicted, Missense 1 and 2 low-sorted subpopulations remained in the low-expression state throughout this experiment, indicating rapid takeover by genomic mutations conferring drug resistance. Accordingly, the corresponding high-sorted subpopulations generated bimodal distributions that became unimodal only after a few days as expected.

As opposed to Missense 1 and 2, Missense 3 low-sorted subpopulations transiently gave rise to new high-expressor subpopulations (Fig. 6 $A-C$ ), indicating either that basal expressionelevating mutants spread much more slowly than in Missense 1 and 2, or that revertant strains arose. For low-sorted Missense 3 replicates $\mathrm{r} 2$ and $\mathrm{r} 3$, the high-expressor peak gradually diminished within $\sim 9 \mathrm{~d}$. In contrast, low-sorted Missense $3 \mathrm{r} 1$ remained stably bimodal from day 5 throughout the end of this 12-d postsort experiment (Fig. $6 C$ and $F$ ).

High-sorted subpopulations from all 3 Missense 3 replicates gave rise to low-expressor cells within $1 \mathrm{~d}$ after flow sorting. Eventually, the high-expressor peak diminished in all high-sorted subpopulations, except as above, for Missense $3 \mathrm{r}$, which
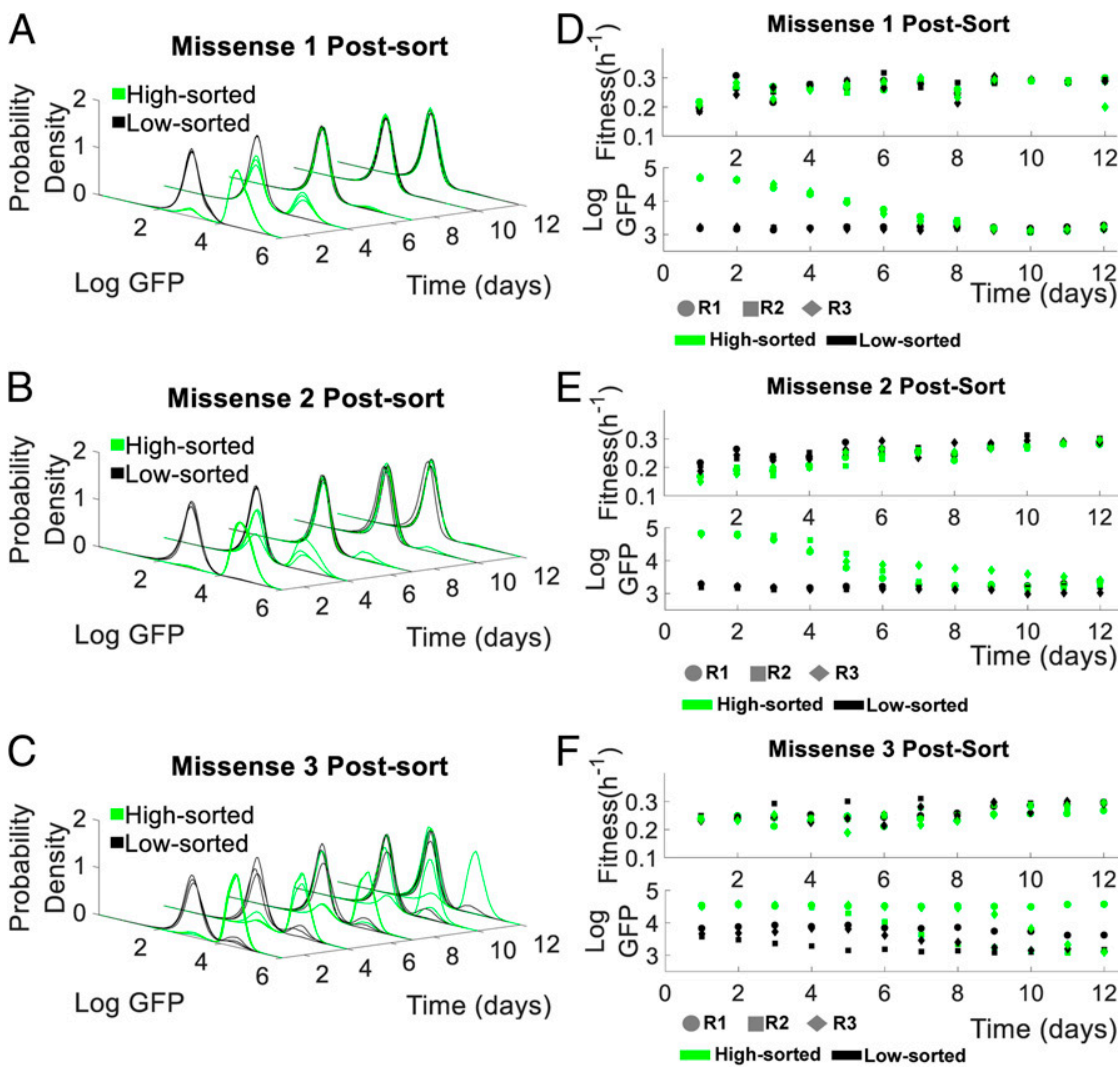

Fig. 6. Postsort evolutionary dynamics of low-sorted and high-sorted subpopulations. (A-C) Histograms of $y E G F P:: z e o R$ expression for Missense 1 , Missense 2 , and Missense 3 low-sorted (black) and high-expressor (green) subpopulations. (D-F) Population fitness and mean $y E G F P:: z e o R$ expression of Missense 1, Missense 2, and Missense 3 low-sorted (black) and high-sorted (green) subpopulations over the course of $12 \mathrm{~d}$. 
remained stably bimodal, biased toward the high-expression state for the last $7 \mathrm{~d}$ postsort (Fig. $6 A-C$ ). Therefore, some Missense 3 r1 clones must be revertant strains with stable high expression.

To identify PF circuit genetic changes underlying these phenotypes, we Sanger-sequenced the $y E G F P:: z e o R$ and $r t T A$ regions of 5 such individual revertant clones. Once again, the PF sequence in revertant clones was genetically identical to the original Missense 3 mutant. WGS (Dataset S3) revealed $1 \mathrm{mu}-$ tation in the SSN2 gene that spread in the high-sorted Missense 3 r1 population over time (reaching $62.2 \%$ on day 12 postsort), suggesting that it confers a survival benefit and is associated with the stable high-expression peak in this population. This mutation was also present at lower frequencies $(5.6 \%$ and $6.6 \%)$ in the respective unsorted and low-sorted populations, further supporting its association with functional reversion. The SSN2 gene encodes an RNA polymerase II mediator complex subunit essential for transcriptional regulation, resembling the general transcriptional-regulatory function of mutations in Missense 2 and 3 r3 (SI Appendix, Tables S3 and S5). However, the dynamic consequences of such gene-expression increases depend on the original mutation (i.e., whether it shrinks or right-shifts the sigmoidal rtTA-synthesis function).

If fully functional revertant strains arose from Missense 3, they should be clearly bimodal in D2Z0, without Zeocin. Thus, to further characterize revertant strains, we studied the yEGFP::zeoR fluorescence distribution in 5 individual Missense $3 \mathrm{r} 1$ clones at the end of the postsort experiment (on day 12) in D2Z2, D0Z2, $\mathrm{D} 2 \mathrm{Z} 0$, and D0Z0 for $4 \mathrm{~d}$. Based on these experiments, Missense 3 r1 revertant clones fell into 2 phenotypic categories: Some had approximately equal peak heights in D2Z2 and D2Z0, whereas other clonal populations consisted almost exclusively of high expressors (Fig. $7 A$ and $B$ and SI Appendix, Fig. S11) like the ancestral PF. Remarkably, the mutations conferred stable high expression even without Zeocin. Additionally, maximum expression shifted leftward, causing the peaks to approach each other compared to the ancestral PF, as predicted by sigmoid elevation in the SI Appendix model (SI Appendix, Mathematical Modeling). Remarkably, the distributions were very similar in $\mathrm{D} 2 \mathrm{Z} 2$ and D2Z0, indicating that the revertant strains were drug resistant at all expression levels (SI Appendix, Fig. S14), so Zeocin did not select for high expression.

\section{Discussion}

Loss-of-function is widely observed in experimental evolution studies $(5,8,13-16)$, presumably because of the large supply of mutations having these effects. While it is always difficult to extrapolate from the laboratory to natural environments, these observations suggest that loss-of-function may be a common mode of adaptation to a new environment. This raises the question of how populations could regain such lost biological functions when the environment changes back to a prior state where that function conferred a selective advantage. This question also has great practical significance for synthetic biology, where we may be able to use evolution to resurrect evolutionarily broken synthetic biological systems in medical, environmental, or extraterrestrial applications where direct human intervention is difficult or possibly ineffective.

To this end, we investigated evolutionary reversibility by evolving 7 yeast strains with broken PF gene circuits, each with a different rtTA mutation, in conditions where regaining gene circuit function would be beneficial. The results revealed various classes of evolutionary dynamics for quasifunctional, dysfunctional, or nonfunctional mutants (Fig. $7 C$ ). Revertant clones arose only in dysfunctional mutant populations through extracircuit mutations. After evolving the broken PF mutants, we found no compensatory coding-sequence mutations inside the gene circuits in any populations. Instead, many new extracircuit mutations increased yEGFP::ZeoR levels, thereby improving drug resistance,
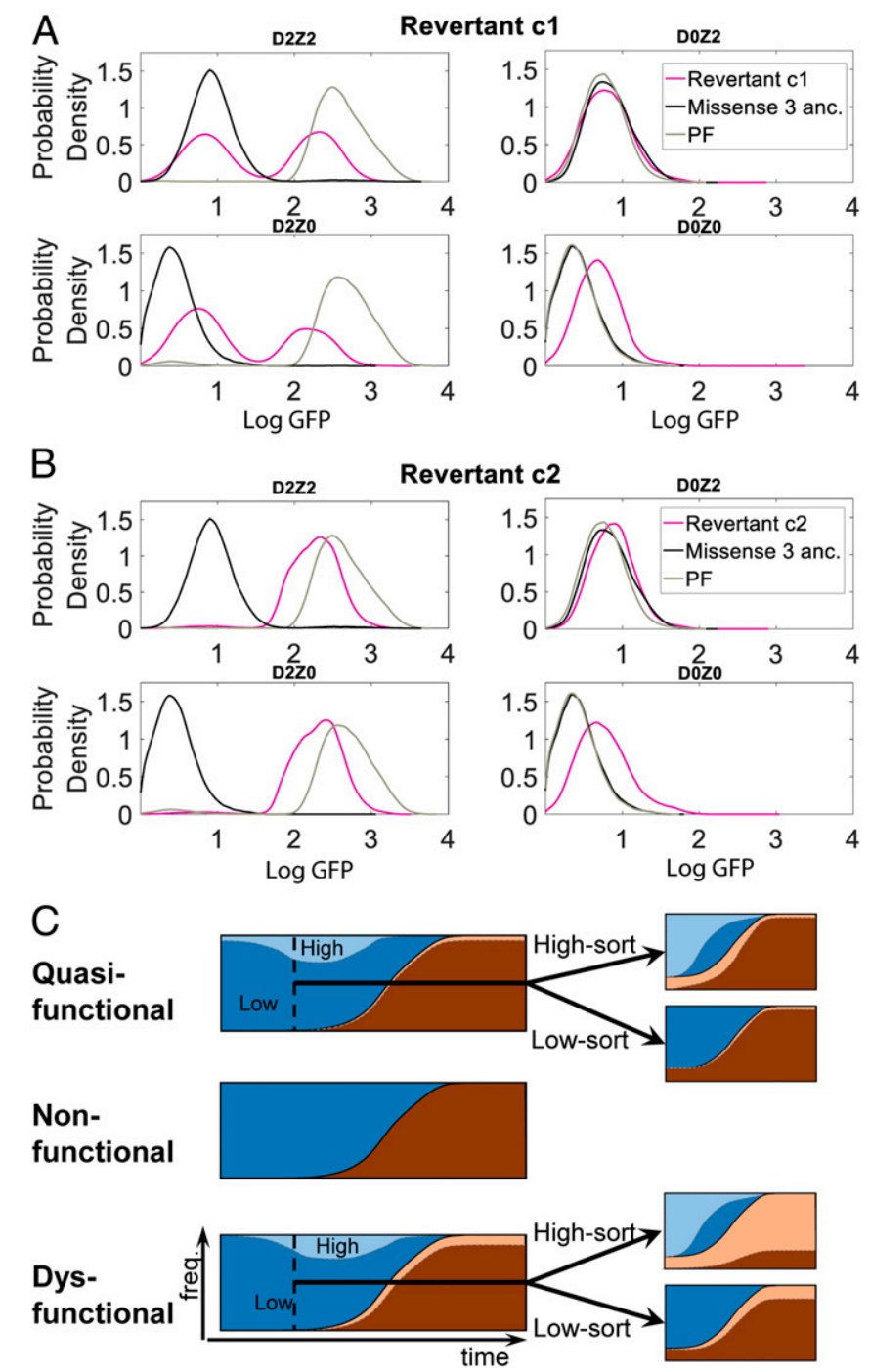

Fig. 7. Phenotypic characterization of revertant clones. $(A)$ Clone 1 from day 12 of high-sorted Missense $3 \mathrm{r} 1$ populations is a representative of revertant clones with approximately equal gene-expression peaks in D2Z2 and D2Z0. (B) Clone 2 from day 12 of high-sorted Missense 3 r1 populations is a representative of Missense 3 clones with predominantly high expressors. Histograms were recorded after maintaining each population over $4 \mathrm{~d}$ in each indicated condition. (C) Muller plot cartoons illustrating schematically time-dependent phenotypic and genotypic frequencies (evolutionary dynamics) during pre- and postsort experimental evolution for quasifunctional, nonfunctional, and dysfunctional PF mutants. Different colors indicate different genotypes. Different shades of the same color indicate different phenotypes associated with the same genotype in bistable populations, the lighter shade corresponding to high expression. The plots ignore phenotypically equivalent competing genotypes for simplicity. For the dysfunctional mutant Missense 3 the plots illustrate only the revertant clone.

without restoring broken PF function, indicating that evolution adopts alternate paths if they are available $(49,50)$. Restricting such alternate paths, e.g., by using higher drug concentrations or by preventing extracircuit genomic mutations (51) may facilitate functional reversions in future experiments. Importantly, some extracircuit mutations did reenable PF gene circuit function by elevating rtTA expression, indicating that evolutionary reversion is possible without any new mutations in rtTA or even the gene circuit. This is broadly consistent with another recent study that evolved Escherichia coli with loss-of-function mutations in a 
particular enzyme, showing some direct revertant mutations in the functional locus but also indirect adaptations elsewhere in the genome (52).

We discovered interesting interactions between intracellular gene circuit dynamics, population dynamics, and evolutionary dynamics (53). Specifically, slow growth rate was not only a fitness parameter, but also a potential enabler of dynamic shifts leading to beneficial early phenotypic and later genetic changes. This is reminiscent of the emerging concept of growth rate as a global regulator of the cell's transcriptional state (54, 55). The potential implications are remarkable, since a slowgrowing dysfunctional mutant can rapidly develop a large highexpression peak, appearing to be reverted. However, these early expression shifts are not truly evolutionary reversions, they simply result from gaining access to a bistable regime that existed for the entire time but was inaccessible through hyperinduction. Indeed, other conditions that decelerate growth similarly to Zeocin, ethanol, and Cisplatin could also boost bistability for such mutants. To combat drug resistance, it will be important to understand how often similar growth-related dynamic shifts $(38,39$, 54) can happen in natural networks of bacteria, yeasts, and mammalian cells. Furthermore, the antibiotic's mechanism of action matters for survival and evolution. As the mathematical model suggested, stressors that reduce growth without directly interfering with protein synthesis enabled access to a bistable regime. On the other hand, antibiotics such as G418 that directly inhibit translation (43) might prevent protein expression-dependent dynamical shifts, and consequently the emergence of high-expressors. Future studies evaluating the effect of different antibiotic mechanisms of action on gene-network dynamics and evolution will be important and informative.

Sanger sequencing revealed only 1 intracircuit mutation across all different tested mutants: A 42-bp deletion eliminating the first tetO2 operator site upstream of the $y E G F P:: z e o R$ coding region. The other tetO2 site upstream from $y E G F P$ ::zeoR and the 2 tetO2 sites upstream from rtTA remained intact, leaving positive feedback unaltered while still enabling $y E G F P:: z e o R$ activation by rtTA. We could not link the observed phenotypes and the identified tetO2 site deletion specifically, except for Missense 3, where 1 tetO2 site was absent only in monostable populations, while bistable clones had intact gene circuits. Notably, tetO2 site deletions occurred in nonfunctional mutants and also in cells previously evolved in D0Z2 (9), suggesting that these deletions contribute to elevating basal yEGFP::zeoR expression independently of rtTA. Establishing the functional and dynamic effects of single versus double tetO2 sites in front of $y E G F P:: z e o R$ requires further investigation. Extracircuit mutations in various genes, such as $S R B 8, M E D 6$, $C C T 7, N U P 159$, and others suggest alterations in the transcription and protein-folding processes, which will require further studies.

Upon sorting and separately culturing low- and high-expressor subpopulations from evolving populations with quasifunctional and dysfunctional mutants, only the latter contained revertant clones that reestablished and maintained bistability. Hence, we successfully generated fully revertant clones capable of stably reactivating the mutant PF circuit. Interestingly, the reversion could be linked to an extracircuit mutation in the SSN2 gene, pointing to functional alterations in the RNA polymerase II-mediator complex controlling transcriptional regulation, and consequently conferring drug resistance through rtTA and yEGFP::zeoR protein levels. Compared to the ancestral PF and the original Missense 3 gene circuit, the revertant strains were Zeocin-resistant (SI Appendix, Fig. S10) yet high expression was not costly, indicating that breaking and then recovering the gene circuit function resulted in PF cell lines with functional gene circuits that are more robust to evolution and environmental perturbations.
Fusing an antibiotic resistance cassette to genes of interest would likely be useful to restore the function of other activator-based gene circuits. The regulators of gene circuits employing only repressors (such as the toggle switch) would be less likely to degrade by evolution in eukaryotes where steric repressors tend to be less toxic than activators, so the evolutionary pressure to mutate them would be lower. Nonetheless, fusing an antibiotic resistance gene to target genes and applying drug selection should allow regaining of function even for repressor-based gene circuits if they break down. Moreover, auxotrophic positive- and negative-selection markers (such as URA3) could be similarly employed as handles for evolutionary restoration, with the advantage that selection could be applied both for low and high expression in yeast.

As opposed to our method, directed evolution studies in the past optimized enzymes and metabolic pathways $(33,34)$ to avoid the difficulties of rational troubleshooting and screening. Such studies focused on individual proteins, mutagenizing coding sequences and screening for optimal variants. In particular, a study titled "Directed evolution of a genetic circuit" in fact mutagenized the coding sequence of a single transcription factor and then screened for optimal variants without actually performing network evolution (33), that is, allowing the entire network and host genome to change naturally. Other studies investigated evolutionary degradation of gene circuits but did not seek to recover or improve gene circuit function by experimental evolution $(9,30)$.

To conclude, our results highlight the versatility of drugresistance mechanisms, including dynamical consequences of slow growth, and exemplify how yeast and possibly mammalian (23) gene circuit evolution can reveal interesting dynamical and biological behaviors, such as host-genomic mutations repairing lost gene circuit function.

\section{Materials and Methods}

We used previously evolved (9) YPH500 haploid S. cerevisiae clones ( $\alpha$, ura3-52, lys2-801, ade2-101, trp1 $\Delta 63$, his3 $\Delta 200$, leu2 $\Delta 1$; Stratagene) with 7 different rtTA-mutant PF synthetic gene circuits (6) stably integrated into chromosome XV near the HIS3 locus (SI Appendix, Table S1). Cells were grown in SD-his-trp $+2 \%$ galactose media at $30{ }^{\circ} \mathrm{C}$, shaking at $300 \mathrm{rpm}$.

To phenotypically characterize the 7 mutants, we performed hysteresis and hyperinduction experiments as well as slow growth rate experiments using $40 \mu \mathrm{g} / \mathrm{mL}$ Cisplatin, $40 \mu \mathrm{g} / \mathrm{mL}$ G418 or $7.5 \%$ ethanol.

To conduct the experimental evolution, $10^{5}$ cells were resuspended into $1 \mathrm{~mL}$ of D2Z0 ( $2 \mu \mathrm{g} / \mathrm{mL}$ Dox) and D2Z2 ( $2 \mu \mathrm{g} / \mathrm{mL}$ Dox, $2 \mathrm{mg} / \mathrm{mL}$ Zeocin) conditions (3 replicates each) every $\sim 24 \mathrm{~h}$. Fluorescence (BD Accuri) and cell counts (Nexcelom) were measured daily. The experiment lasted for $14 \mathrm{~d}$. Missense 1 , 2 , and 3 populations were FACS-sorted (BD FACSAria III) at day 4. Collected low- and high-expressor subpopulations were cultured separately in D2Z2 for $12 \mathrm{~d}$. Flow-cytometry (BD Accuri) and cell counts (Nexcelom) were performed daily on the sorted samples.

To assess the fitness of evolved populations in Zeocin, we collected OD600 readings in D0Z2 over $24 \mathrm{~h}$. We genetically characterized evolution endpoint populations and clonal isolates through WGS and Sanger sequencing, respectively. Evolved clonal isolates were phenotyped in D2Z2. Revertant clonal isolates were phenotyped in D0Z0, D2Z0, and D0Z2, in addition to D2Z2.

Detailed protocols are included in the SI Appendix, Supporting Materials and Methods.

Data Availability. Data and Matlab code associated with the figures (56) and raw sequencing data (57) can be found at https://openwetware.org/wiki/ CHIP:Data.

ACKNOWLEDGMENTS. We thank Rebecca C. Connor and Todd Rueb for their help in performing the FACS sorting and the flow-cytometry readings at the Stony Brook flow cytometry facility; Oleksandra Romanyshyn and Michael Tyler Guinn for facilitating the timely execution of experiments; 2 anonymous reviewers for their insightful comments; and all G.B. laboratory members for helpful discussions and feedback. This work was supported by the NIH/National Institute of General Medical Sciences Maximizing Investigator's Research Award (MIRA) Grant R35 GM122561 (to G.B.), the Laufer Center for Physical and Quantitative Biology (G.B.), and the Swiss National Science Foundation Ambizione Grant PZ00P3_180147 (to M.M.). 
1. E. Kussell, S. Leibler, Phenotypic diversity, population growth, and information in fluctuating environments. Science 309, 2075-2078 (2005).

2. D. M. Wolf, V. V. Vazirani, A. P. Arkin, Diversity in times of adversity: Probabilistic strategies in microbial survival games. J. Theor. Biol. 234, 227-253 (2005).

3. J. A. Hill, T. R. O'Meara, L. E. Cowen, Fitness trade-offs associated with the evolution of resistance to antifungal drug combinations. Cell Rep. 10, 809-819 (2015).

4. M. Eames, T. Kortemme, Cost-benefit tradeoffs in engineered lac operons. Science 336, 911-915 (2012)

5. G. I. Lang, A. W. Murray, D. Botstein, The cost of gene expression underlies a fitness trade-off in yeast. Proc. Natl. Acad. Sci. U.S.A. 106, 5755-5760 (2009).

6. D. Nevozhay, R. M. Adams, E. Van Itallie, M. R. Bennett, G. Balázsi, Mapping the environmental fitness landscape of a synthetic gene circuit. PLoS Comput. Biol. 8 e1002480 (2012).

7. T. Kalisky, E. Dekel, U. Alon, Cost-benefit theory and optimal design of gene regu lation functions. Phys. Biol. 4, 229-245 (2007).

8. T. F. Cooper, D. E. Rozen, R. E. Lenski, Parallel changes in gene expression after 20,000 generations of evolution in Escherichiacoli. Proc. Natl. Acad. Sci. U.S.A. 100, 1072 1077 (2003).

9. C. González et al., Stress-response balance drives the evolution of a network module and its host genome. Mol. Syst. Biol. 11, 827 (2015).

10. E. Dekel, U. Alon, Optimality and evolutionary tuning of the expression level of a protein. Nature 436, 588-592 (2005).

11. L. Tan, S. Serene, H. X. Chao, J. Gore, Hidden randomness between fitness landscapes limits reverse evolution. Phys. Rev. Lett. 106, 198102 (2011).

12. J. J. Kuzdzal-Fick, L. Chen, G. Balázsi, Disadvantages and benefits of evolved unicellularity versus multicellularity in budding yeast. Ecol. Evol. 9, 8509-8523 (2019).

13. A. K. Hottes et al., Bacterial adaptation through loss of function. PLoS Genet. 9, e1003617 (2013).

14. G. I. Lang, M. M. Desai, The spectrum of adaptive mutations in experimental evolution. Genomics 104, 412-416 (2014)

15. Y.-C. Xu et al., Adaptation and phenotypic diversification in Arabidopsis through loss of-function mutations in protein-coding genes. Plant Cell 31, 1012-1025 (2019).

16. M. J. Behe, Experimental evolution, loss-of-function mutations, and "the first rule of adaptive evolution". Q. Rev. Biol. 85, 419-445 (2010)

17. N. Philippe, E. Crozat, R. E. Lenski, D. Schneider, Evolution of global regulatory networks during a long-term experiment with Escherichia coli. BioEssays 29, 846-860 (2007).

18. S. Quan et al., Adaptive evolution of the lactose utilization network in experimentally evolved populations of Escherichia coli. PLoS Genet. 8, e1002444 (2012).

19. C. Igler, M. Lagator, G. Tkačik, J. P. Bollback, C. C. Guet, Evolutionary potential of transcription factors for gene regulatory rewiring. Nat. Ecol. Evol. 2, 1633-1643 (2018)

20. M. Isalan et al., Evolvability and hierarchy in rewired bacterial gene networks. Nature 452, 840-845 (2008)

21. F. J. Poelwijk, M. G. J. de Vos, S. J. Tans, Tradeoffs and optimality in the evolution of gene regulation. Cell 146, 462-470 (2011).

22. Z. Bódi et al., Phenotypic heterogeneity promotes adaptive evolution. PLoS Biol. 15 e2000644 (2017).

23. K. S. Farquhar et al., Role of network-mediated stochasticity in mammalian drug resistance. Nat. Commun. 10, 2766 (2019)

24. T. S. Bayer, Using synthetic biology to understand the evolution of gene expression Curr. Biol. 20, R772-R779 (2010)

25. T. S. Gardner, C. R. Cantor, J. J. Collins, Construction of a genetic toggle switch in Escherichia coli. Nature 403, 339-342 (2000)

26. M. B. Elowitz, S. Leibler, A synthetic oscillatory network of transcriptional regulators. Nature 403, 335-338 (2000).

27. A. Becskei, L. Serrano, Engineering stability in gene networks by autoregulation. Nature 405, 590-593 (2000)

28. C. J. Bashor, J. J. Collins, Understanding biological regulation through synthetic biology. Annu. Rev. Biophys. 47, 399-423 (2018)

29. G. M. Church, M. B. Elowitz, C. D. Smolke, C. A. Voigt, R. Weiss, Realizing the potential of synthetic biology. Nat. Rev. Mol. Cell Biol. 15, 289-294 (2014).

30. S. C. Sleight, B. A. Bartley, J. A. Lieviant, H. M. Sauro, Designing and engineering evolutionary robust genetic circuits. J. Biol. Eng. 4, 12 (2010).

31. F. Wu, D. J. Menn, X. Wang, Quorum-sensing crosstalk-driven synthetic circuits: From unimodality to trimodality. Chem. Biol. 21, 1629-1638 (2014).
32. T. Ellis, X. Wang, J. J. Collins, Diversity-based, model-guided construction of synthetic gene networks with predicted functions. Nat. Biotechnol. 27, 465-471 (2009)

33. Y. Yokobayashi, R. Weiss, F. H. Arnold, Directed evolution of a genetic circuit. Proc. Natl. Acad. Sci. U.S.A. 99, 16587-16591 (2002)

34. C. Schmidt-Dannert, D. Umeno, F. H. Arnold, Molecular breeding of carotenoid biosynthetic pathways. Nat. Biotechnol. 18, 750-753 (2000)

35. R. P. Bennett, C. A. Cox, J. P. Hoeffler, Fusion of green fluorescent protein with the Zeocin-resistance marker allows visual screening and drug selection of transfected eukaryotic cells. Biotechniques 24, 478-482 (1998).

36. A. Gatignol, H. Durand, G. Tiraby, Bleomycin resistance conferred by a drug-binding protein. FEBS Lett. 230, 171-175 (1988).

37. M. Oliva-Trastoy, M. Defais, F. Larminat, Resistance to the antibiotic Zeocin by stable expression of the Sh ble gene does not fully suppress Zeocin-induced DNA cleavage in human cells. Mutagenesis 20, 111-114 (2005).

38. J. B. Deris et al., The innate growth bistability and fitness landscapes of antibioticresistant bacteria. Science 342, 1237435 (2013).

39. C. Tan, P. Marguet, L. You, Emergent bistability by a growth-modulating positive feedback circuit. Nat. Chem. Biol. 5, 842-848 (2009).

40. S. Dasari, P. B. Tchounwou, Cisplatin in cancer therapy: Molecular mechanisms of action. Eur. J. Pharmacol. 740, 364-378 (2014).

41. D. C. Eustice, J. M. Wilhelm, Mechanisms of action of aminoglycoside antibiotics in eucaryotic protein synthesis. Antimicrob. Agents Chemother. 26, 53-60 (1984).

42. R. L. Adams, L. J. Terry, S. R. Wente, Nucleoporin FG domains facilitate mRNP remodeling at the cytoplasmic face of the nuclear pore complex. Genetics 197, 12131224 (2014).

43. T. Borggrefe, R. Davis, H. Erdjument-Bromage, P. Tempst, R. D. Kornberg, A complex of the Srb8, $-9,-10$, and -11 transcriptional regulatory proteins from yeast. J. Biol. Chem. 277, 44202-44207 (2002).

44. Y. C. Lee, S. Min, B. S. Gim, Y. J. Kim, A transcriptional mediator protein that is required for activation of many RNA polymerase II promoters and is conserved from yeast to humans. Mol. Cell. Biol. 17, 4622-4632 (1997)

45. Y. Takagi, R. D. Kornberg, Mediator as a general transcription factor. J. Biol. Chem. 281, 80-89 (2006).

46. V. Stoldt et al., Review: The Cct eukaryotic chaperonin subunits of Saccharomyces cerevisiae and other yeasts. Yeast 12, 523-529 (1996).

47. M. M. Luke et al., The SAP, a new family of proteins, associate and function positively with the SIT4 phosphatase. Mol. Cell. Biol. 16, 2744-2755 (1996).

48. C. Cappellaro, V. Mrsa, W. Tanner, New potential cell wall glucanases of Saccharomyces cerevisiae and their involvement in mating. J. Bacteriol. 180, 5030-5037 (1998).

49. S. Kim, T. D. Lieberman, R. Kishony, Alternating antibiotic treatments constrain evolutionary paths to multidrug resistance. Proc. Natl. Acad. Sci. U.S.A. 111, 1449414499 (2014)

50. F. J. Poelwijk, D. J. Kiviet, D. M. Weinreich, S. J. Tans, Empirical fitness landscapes reveal accessible evolutionary paths. Nature 445, 383-386 (2007).

51. A. Chavez et al., Precise Cas9 targeting enables genomic mutation prevention. Proc. Natl. Acad. Sci. U.S.A. 115, 3669-3673 (2018).

52. J. V. Rodrigues, E. I. Shakhnovich, Adaptation to mutational inactivation of an essential gene converges to an accessible suboptimal fitness peak. elife 8, e50509 (2019)

53. A. Sanchez, J. Gore, Feedback between population and evolutionary dynamics determines the fate of social microbial populations. PLoS Biol. 11, e1001547 (2013)

54. A. Y. Weiße, D. A. Oyarzún, V. Danos, P. S. Swain, Mechanistic links between cellular trade-offs, gene expression, and growth. Proc. Natl. Acad. Sci. U.S.A. 112, E1038E1047 (2015)

55. M. Scott, C. W. Gunderson, E. M. Mateescu, Z. Zhang, T. Hwa, Interdependence of cell growth and gene expression: Origins and consequences. Science 330, 10991102 (2010)

56. M. K. Gouda, G. Balázsi, Flow cytometry and cell count data. Google Drive. https:// drive.google.com/drive/folders/13jrR8XHP4p96-bcNGTIxRpFzjTfRvPkh. Deposited 16 October 2019.

57. M. K. Gouda, G. Balázsi, Raw whole-genome sequencing (WGS) data. Google Drive. https://drive.google.com/drive/folders/1sZxfXY-AzigYSCDhX_SWObSNqfgo72lh. Deposited 16 October 2019. 